further testing proved that the transmission of sounds was not suffciently perfect for practical purposes.

I next proposed trying the addition of the microphone, and for this purpose Dr. Wallich had kindly offered me the use of his instrument and his valuable assistance; but I now believe that I have been forestalled by Mr. Rhodes, although I have not had an opportunity of seeing his instrument yet.

The following simple experiment may be tried by anyone, and will illustrate the principle on which both Graydon's audiphone and mine are intended to work. Take an ordinary toy telephone, consisting of a drum and string attached to the centre of the membrane; hold the string in the teeth; and, with this duly stretched, let some one speak into the drum. Now, if the ears be closed by the fingers, the conversation will be heard through the skull.

Of course, we have yet to see whether these instruments will prove of practical value; but I feel convinced that it is by working in this direction that we may hope to obtain that universal desideratum, spectacles for the deaf.

\section{THE ANTISEPTIC TREATMENT OF PHTHISIS PULMONALIS.}

By G. HUNTER MACKENZIE, M.D.,

Lecturer on Diseases of the Throat in the School of Medicine, Edinburgh.

THE article by Dr. Burney Yeo in the Journal of the 20 th of December last induces me to bring forward a method of treating ordinary inflammatory phthisis, from which I have obtained good results. Having, some months ago, found bacteric organisms in the sputum of a phthisical patient, it occurred to me whether their presence might be associated with a process of blood-poisoning from the decomposition and putrefaction of the products of inflammation in the pulmonary bronchi and air-cells. I accordingly experimented with various antiseptic inhalations of different strengths, and latterly have been using pure carbolic acid or creasote. I have had made, by Mr. Young of this city, a respirator somewhat resembling that of Dr. Roberts of Manchester, but which covers both mouth and nose, and has a double breathing. chamber for containing pieces of sponge saturated with a strong carbolic or creasote solution. The sponge is covered with a light layer of cotton-wool, to act as an additional filter and prevent the outward evaporation of the antiseptic. The respirator is worn as continuously as possible both by night and day. By this means, not only is the respired air purified, but the volatile antiseptic is drawn in with each inspiration and thoroughly permeates the lungs. A renewed application of the fluid is made night and morning by means of a camel's hair brush. I have had no difficulty in inducing even young children to wear the respirator for lengthened periods, the only drawback having been a tendency to chafing of the skin by the continuous pressure of the borders of the instrument. This, however, has been overcome by having the instrument bordered with soft sponge, which answers admirably. My experience of this method of treatment has as yet been limited, and confined to cases in their first stage. The results obtained, however, have been so satisfactory as to justify me in discarding the internal administration of the various specifics and continuing the inhalations alone. It is, of course, impossible for me to say that cases of phthisis are absolutely cured by this system of treatment; but the distressing symptoms of phthisis - the night-sweats, cough, and impaired appetite -have been so ameliorated as to justify me in publishing this preliminary note, reserving for a tuture communication further details of the method and notes of the cases tried by it.

\section{A CASE OF "CEREBRAL PNEUMONIA." \\ BY JAMES RUSSELL; M.D., F.R.C.P., Physician to the Birmingham General Hospital.}

A CASE which was under my care in the Birmingham General Hospital is worth notice, as illustrating two points of considerable interest in the natural history of pneumonia; the former relating to the diagnosis, the latter to the pathology of the disease. First, the case was one in which the nervous disturbance at the outset was unusually prominent and severe, "so as in some cases, and especially with children, to divert attention from the pulmonary condition". In Dr. Sturges' able work on Pneumonia, from which the preceding quotation is made, other interesting illustrations are given.* The second particular is that high pyrexia continued through four days, before pulmonary symptoms made

* I might refer also to observations of a similar character in relation to the onset of cardiac inflammation, given by Dr. Latham in his Clinical Lectures on Diseases of the Heart. their appearance. Some important remarks on this phenomenon, not altogether rare in pneumonia, are made by Dr. Sturges, especially in two notes, pp. 33 and 168 ; and the value of the observation in relation to the theory of the disease is made apparent in the same volume.

It is worth noting, in connection with the diagnosis, that not only were physical and general signs of pneumonia absent during these four days, but that respiration was hardly quickened until the local signs were developed. I would observe, with respect to the cerebral symptom, that, although the pain in the head was most severe, and the vomiting constant, the boy exhibited a striking contrast with a case of meningitis in the same ward. The latter boy was found lying in bed, coiled up under the clathing, and intolerant of interference; the instant the examination was ended, he was again beneath the covering, with the rapidity of a spring released. My present patient had much more urgent pain and vomiting, but comparatively little intolerance of light, and was not much troubled by examination.

The boy was ten years of age. He shivered one morning, having been previously in good health; was dull during the day, complained of headache, and vomited. When he was brought to the hospital the same evening, his temperature was $103.6^{\circ}$, and $105^{\circ}$ at midnight. In the course of the following day, the temperature dropped to $102^{\circ}$, but began again to rise, reaching $104^{\circ}$ on the fourth morning. Meantime, the pulse was I20 ; respiration never above 25 . During this time, the boy had intense headache, and constant vomiting of green fluid. There were neither physical nor general symptoms of chest-affection, though attention was carefully directed to the condition of the lungs. There was a slight eruption of herpes at one angle of the mouth. On the fourth day, some extension of splenic dulness was observed ; the cerebral symptoms were partially relieved. Next morning, general consolidation was discovered of the right lower lobe. The pulse had risen to $130-140$, the respiration $34-40$; and all symptoms of cerebral disorder had completely cleared away. The remainder of the case was typical of pneumonia ; the temperature beginning to fall in the night of the seventh day of the attack, and reaching the normal standard on the next morning but one. The urine contained from one-eighth to one-fourth of the column of albumen. Recovery was complete and rapid.

\section{THERAPEUTIC MEMORANDA.}

\section{FLITWICK WATER.}

AT the Royal Hospital for Diseases of the Chest, I have been induced to use the natural mineral water of Flitwick, a village about two miles from Ampthill, in Bedfordshire. This water, according to the analysis of Professor W. White, contains in each gallon :

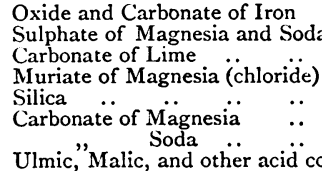

Ulmic,"Malic, and other acid constituents of vegetable matters $\quad . . \quad 0.22$

One of the most marked cases in which benefit accrued from its use was that of a railway signalman, excessively anæmic after protracted hæmoptysis, and with whom all the usual ferruginous preparations disagreed. I have also notes of six other hospital patients : two debilitated after acute rheumatism, where the perchloride of iron caused headache and constipation; and four chlorotic females, on whom this potent chalybeate acted beneficially when the usual iron medicines were not well borne. I hope this note may lead my professional brethren to give this water a trial. $\quad$ G. GODDARD ROGERS, M.D.

\section{BLOODLETTING IN HEADACHE WITH INSOMNIA DUE} TO CONGESTION OF THE BRAIN.

WirHin two years, I have treated three cases of headache associated with intractable sleeplessness, in each of which bloodletting was followed by speedy and effectual cure.

CASE I. - Mrs. A., aged about 30, complained of headache and sleeplessness. The headache was always worse when lying down. The subconjur.ctival vessels were much congested. For four weeks, various internal and external remedies were tried in vain, such as the iodide and bromide of potassium, blisters, hot and cold fomentations, etc. To procure sleep, I prescribed, with very little benefit, large doses of chloral and other hypnotics. Failing to give relief, I suggested bleeding. I opened the right cephalic vein, and drew off in a full stream about fifteen ounces, when the patient said that her headache had quite gone, and that she felt very comfortable. About twenty ounces were 